\title{
AN ALGORITHM FOR CONSTRUCTING BIORTHOGONAL MULTIWAVELETS WITH HIGHER APPROXIMATION ORDERS
}

\author{
YANG SHOUZHI ${ }^{1}$
}

(Received 11 November, 2004; revised 24 February, 2006)

\begin{abstract}
Given a pair of biorthogonal multiscaling functions, we present an algorithm for raising their approximation orders to any desired level. Precisely, let $\Phi(x)$ and $\tilde{\Phi}(x)$ be a pair of biorthogonal multiscaling functions of multiplicity $r$, with approximation orders $m$ and $\tilde{m}$, respectively. Then for some integer $s$, we can construct a pair of new biorthogonal multiscaling functions $\Phi^{\text {new }}(x)=\left[\Phi^{T}(x), \phi_{r+1}(x), \phi_{r+2}(x), \ldots, \phi_{r+s}(x)\right]^{T}$ and $\tilde{\Phi}^{\text {new }}(x)=$ $\left[\tilde{\Phi}(x)^{T}, \tilde{\phi}_{r+1}(x), \bar{\phi}_{r+2}(x), \ldots, \tilde{\phi}_{r+s}(x)\right]^{T}$ with approximation orders $n(n>m)$ and $\tilde{n}$ $(\tilde{n}>\tilde{m})$, respectively. In addition, corresponding to $\Phi^{\text {new }}(x)$ and $\bar{\Phi}^{\text {new }}(x)$, a biorthogonal multiwavelet pair $\Psi^{\text {new }}(x)$ and $\tilde{\Psi}^{\text {new }}(x)$ is constructed explicitly. Finally, an example is given.
\end{abstract}

2000 Mathematics subject classification: primary 42C15, $94 \mathrm{~A} 12$.

Keywords and phrases: biorthogonal, multiscaling functions, multiwavelets, approximation order.

\section{Introduction}

A refinable function vector of multiplicity $r$ is a vector $\Phi(x)=\left[\phi_{1}(x), \ldots, \phi_{r}(x)\right]^{T}$, which satisfies a matrix refinement equation

$$
\Phi(x)=\sum_{k} P_{k} \Phi(2 x-k) .
$$

The sequence $\left\{P_{k}\right\}_{k \in Z}$ of coefficient matrices is called the two-scale matrix sequence of $\Phi(x)$. We assume that only finitely many $P_{k}$ are nonzero and that all $\phi_{j}(x)$ have compact support.

We call $\Phi(x)$ a multiscaling function with multiplicity $r$ if it generates a multiresolution analysis (MRA) of $L^{2}(R)$. This means that there exists a sequence of subspaces $V_{j}, j \in Z$, of $L^{2}(R)$ with the following properties:

\footnotetext{
'Department of Mathematics, Shantou University, Shantou 515063, P. R. China; e-mail: szyang@stu.edu.cn.

(C) Australian Mathematical Society 2006, Serial-fee code 1446-1811/06
} 
(1) $\cdots \subset V_{0} \subset V_{1} \subset V_{2} \cdots$;

(2) $\operatorname{clos}_{L^{2}(R)}\left(\bigcup_{j \in Z} V_{j}\right)=L^{2}(R)$;

(3) $\bigcap_{j \in Z} V_{j}=\{0\}$

(4) $f(x) \in V_{j} \Leftrightarrow f(2 x) \in V_{j+1}, j \in Z$;

(5) The family $\left\{\phi_{\ell}(x-k): 1 \leq \ell \leq r, k \in Z\right\}$ forms a Riesz basis of $V_{0}$.

In detail, property (5) means that there exist two constants $0<A \leq B<\infty$ so that

$$
A \sum_{j \in Z}\left\|C_{j}\right\|_{2}^{2} \leq\left\|\sum_{j \in Z} C_{j}^{*} \Phi(x-j)\right\|_{2}^{2} \leq B \sum_{j \in Z}\left\|C_{j}\right\|_{2}^{2}
$$

for any sequence of coefficient vectors $\left\{C_{j}\right\}$ with $\sum_{j \in Z}\left\|C_{j}\right\|_{2}^{2}<\infty$. The superscript * denotes the transpose.

Corresponding to a multiscaling function $\Phi(x), \Psi(x)=\left[\psi_{1}(x), \ldots, \psi_{r}(x)\right]^{T}$ is called a multiwavelet if $\left\{\psi_{\ell}(x-k): 1 \leq \ell \leq r ; k \in Z\right\}$ forms Riesz bases of subspace $W_{0}$ so that $V_{1}=V_{0} \oplus W_{0}$ and $\left\{2^{n / 2} \psi_{\ell}\left(2^{n} x-k\right): 1 \leq \ell \leq r ; k, n \in Z\right\}$ forms a Riesz basis of $L^{2}(R)$.

$\Psi(x)=\left[\psi_{1}(x), \ldots, \psi_{r}(x)\right]^{T}$ satisfies the refinement equation

$$
\Psi(x)=\sum_{k \in Z} Q_{k} \Phi(2 x-k)
$$

for some $r \times r$ matrices sequence $\left\{Q_{k}\right\}_{k \in Z}$.

By taking Fourier transforms on both sides of (1.1) and (1.2), respectively, we have

$$
\begin{array}{ll}
\hat{\Phi}(w)=P\left(e^{-i w / 2}\right) \hat{\Phi}(w / 2), & P(z)=\frac{1}{2} \sum_{k \in Z} P_{k} z^{k}, \\
\hat{\Psi}(w)=Q\left(e^{-i w / 2}\right) \hat{\Phi}(w / 2), & Q(z)=\frac{1}{2} \sum_{k \in Z} Q_{k} z^{k},
\end{array}
$$

where $P(z)$ and $Q(z)$ are called the two-scale matrix symbols of $\Phi(x)$ and $\Psi(x)$, respectively.

The properties of multiscaling functions and multiwavelets are discussed in many papers (see $[3,4,6,15,17-19]$ ). One of the properties of a multiscaling function which has great practical interest is the approximation order (see [2, 8, 10-12]). One known way to raise the approximation order is through the use of two-scale similarity transforms (TSTs) (see $[13,16]$ ). In this paper, we will give a general scheme for constructing a pair of biorthogonal multiscaling functions and multiwavelets with arbitrary desired approximation orders from any given pair of biorthogonal multiscaling functions $\Phi(x)$ and $\tilde{\Phi}(x)$. In addition, we also present an explicit formula for constructing a pair of biorthogonal multiwavelets $\Psi^{\text {new }}(x)$ and $\tilde{\Psi}^{\text {new }}(x)$ associated with a new biorthogonal multiscaling function pair $\Phi^{\text {new }}(x)$ and $\tilde{\Phi}^{\text {new }}(x)$. 


\section{Basic concept}

Two multiscaling functions $\Phi(x)$ and $\tilde{\Phi}(x)$ form a biorthogonal pair if

$$
\langle\Phi(x), \tilde{\Phi}(x-k)\rangle=\delta_{0, k} I_{r \times r}, \quad k \in Z,
$$

where $\delta$ is the Kronecker delta, and $I_{r \times r}$ denotes the identity matrix.

Corresponding to $\Phi(x)$ and $\tilde{\Phi}(x)$, two multiwavelets $\Psi(x)=\left[\psi_{1}(x), \ldots, \psi_{r}(x)\right]^{T}$ and $\tilde{\Psi}(x)=\left[\tilde{\psi}_{1}(x), \ldots, \tilde{\psi}_{r}(x)\right]^{T}$ form a biorthogonal multiwavelet pair if they satisfy the following equations:

$$
\begin{aligned}
& \langle\Phi(x), \tilde{\Psi}(x-k)\rangle=\langle\Psi(x), \tilde{\Phi}(x-k)\rangle=O_{r \times r}, \\
& \langle\Psi(x), \tilde{\Psi}(x-k)\rangle=\delta_{0, k} I_{r \times r}, \quad k \in Z,
\end{aligned}
$$

where $O_{r \times r}$ denotes the zero matrix.

Similarly, let $\tilde{P}(z)$ and $\tilde{Q}(z)$ be the two-scale matrix symbols of $\tilde{\Phi}(x)$ and $\tilde{\Psi}(x)$, respectively. In terms of the two-scale matrix symbols $P(z), Q(z), \tilde{P}(z)$ and $\tilde{Q}(z)$, the biorthogonality of conditions (2.1)-(2.2) implies (see $[1,9,19]$ )

$$
\left\{\begin{array}{l}
P(z) \tilde{P}(z)^{*}+P(-z) \tilde{P}(-z)^{*}=I_{r \times r} \\
P(z) \tilde{Q}(z)^{*}+P(-z) \tilde{Q}(-z)^{*}=O_{r \times r} \\
\tilde{P}(z) Q(z)^{*}+\tilde{P}(-z) Q(-z)^{*}=O_{r \times r} \\
Q(z) \tilde{Q}(z)^{*}+Q(-z) \tilde{Q}(-z)^{*}=I_{r \times r}
\end{array}\right.
$$

LEMMA 2.1. Let $\Phi(x)$ and $\tilde{\Phi}(x)$ be a pair of biorthogonal multiscaling functions, and let $\Psi(x)$ and $\tilde{\Psi}(x)$ be the corresponding biorthogonal multiwavelet pair, with two-scale matrix symbols $P(z), \tilde{P}(z), Q(z)$ and $\tilde{Q}(z)$, respectively. Suppose $Q^{k}(z)$, $k=1, \ldots, r$ is the kth row of $Q(z)$, and $\tilde{Q}^{k}(z), k=1, \ldots, r$ is the kth row of $\tilde{Q}(z)$. Then

$$
\left\{\begin{aligned}
P(z) \tilde{Q}^{k}(z)^{*}+P(-z) \tilde{Q}^{k}(-z)^{*} & =O_{r \times 1}, & k & =1, \ldots, r, \\
\tilde{P}(z) Q^{k}(z)^{*}+\tilde{P}(-z) Q^{k}(-z)^{*} & =O_{r \times 1}, & k & =1, \ldots, r, \\
Q^{j}(z) \tilde{Q}^{k}(z)^{*}+Q^{j}(-z) \tilde{Q}^{k}(-z)^{*} & =\delta_{j, k}, & j, k & =1, \ldots, r .
\end{aligned}\right.
$$

PROOF. In terms of the biorthogonality of $\Phi(x), \tilde{\Phi}(x), \Psi(x)$ and $\tilde{\Psi}(x)$, we can show that $P(z), Q(z), \tilde{P}(z)$ and $\tilde{Q}(z)$ satisfy (2.3). Substituting $Q(z)=$ $\left[Q^{1}(z)^{*}, \ldots, Q^{r}(z)^{*}\right]^{*}$ and $\tilde{Q}(z)=\left[\tilde{Q}^{1}(z)^{*}, \ldots, \tilde{Q}^{r}(z)^{*}\right]^{*}$ into (2.3), respectively, we obtain (2.4). 
A multiscaling function $\Phi(x)$ has approximation order $m \geq 1$ if $m$ is the largest integer for which there is a set of row vectors $\left\{\mathbf{a}^{\ell}\right\}_{\ell=0}^{m-1} \subset R^{1 \times r}$, with $\mathbf{a}^{0} \neq O_{1 \times r}$ that satisfy, for $\ell=0,1, \ldots, m-1$,

$$
\begin{array}{r}
\sum_{k=0}^{\ell}(-1)^{k} \frac{1}{2^{k}}\left(\begin{array}{l}
\ell \\
k
\end{array}\right) \mathbf{a}^{\ell-k} \sum_{j \in Z}(2 j)^{k} P_{2 j}=\frac{1}{2^{\ell}} \mathbf{a}^{\ell}, \\
\sum_{k=0}^{\ell}(-1)^{k} \frac{1}{2^{k}}\left(\begin{array}{l}
\ell \\
k
\end{array}\right) \mathbf{a}^{\ell-k} \sum_{j \in Z}(2 j+1)^{k} P_{2 j+1}=\frac{1}{2^{\ell}} \mathbf{a}^{\ell} .
\end{array}
$$

See $[8,10,11]$ for details. As is well known, if a multiscaling function $\Phi(x)$ has approximation order $m$, this implies that the multiwavelet $\tilde{\Psi}(x)$ has $m$ vanishing moments, that is, $\int x^{j} \tilde{\psi}_{k}(x) d x=0$, for $j=0,1, \ldots, m-1 ; k=1, \ldots, r$.

By repeated application of (1.3), we have

$$
\hat{\Phi}(w)=\left(\prod_{j=1}^{\infty} P\left(e^{-i w / 2^{j}}\right)\right) \hat{\Phi}(0) .
$$

According to $[3,5]$, the infinite matrix product $\left(\prod_{j=1}^{\infty} P\left(e^{-i w / 2^{j}}\right)\right)$ converges uniformly on compact sets to a continuous matrix-valued function if and only if $P(1)$ has eigenvalues $\lambda_{1}=\cdots=\lambda_{k}=1$ and $\left|\lambda_{k+1}\right|, \ldots,\left|\lambda_{r}\right|<1$, with the eigenvalue 1 nondegenerate for $k \geq 1$.

A two-scale matrix symbol $P(z)$ satisfies Condition $\mathbf{E}$, if $P(1)$ has a simple eigenvalue of 1 , with all other eigenvalues less than 1 in modulus. Condition $E$ is automatically satisfied if the two-scale matrix symbol $P(z)$ generates an MRA of $L^{2}(R)$ with compactly supported basis functions.

In order to obtain the conditions that the matrix refinement equation has an $L^{2}$-stable solution, we introduce the transition operator $\mathscr{T}_{P}$ :

$$
\mathscr{T}_{P} A\left(z^{2}\right)=P(z) A(z) P(z)^{*}+P(-z) A(-z) P(-z)^{*},
$$

where $A(z)$ is an $r \times r$ matrix with trigonometric polynomial entries. See [15] for details. It was shown in [15] that the matrix refinement equation has an $L^{2}$-stable solution if and only if the corresponding transition operator $\mathscr{T}_{P}$ satisfies Condition $\mathbf{E}$, and its eigenmatrix corresponding to the eigenvalue 1 is positive definite for all $w \in R$.

\section{Biorthogonal multiscaling functions}

In this section, we will introduce a procedure for constructing a pair of biorthogonal multiscaling functions with multiplicity $r+s$ starting with any given pair of biorthogonal multiscaling functions with multiplicity $r$. 
Let $H(z)=\left[h_{i, j}(z)\right]$ be the $s \times r$ matrix of Laurent polynomials with $H(z)=$ $H(-z)$ and $H(z) H(z)^{*}=C l(0<C<1,|z|=1)$. Construct two $s \times r$ matrices $A(z)$ and $\tilde{A}(z)$ as follows:

$$
\begin{aligned}
& A(z)=H(z) Q(z), \\
& \tilde{A}(z)=H(z) \tilde{Q}(z) .
\end{aligned}
$$

LEMMA 3.1. In the setting of Lemma 2.1, suppose that $A(z)$ and $\tilde{A}(z)$ are two $s \times r$ matrices defined in (3.1) and (3.2), respectively. Then

$$
\begin{aligned}
& A(z) \tilde{A}(z)^{*}+A(-z) \tilde{A}(-z)^{*}=C I_{s \times s} \\
& P(z) \tilde{A}(z)^{*}+P(-z) \tilde{A}(-z)^{*}=O_{r \times s}, \\
& \tilde{P}(z) A(z)^{*}+\tilde{P}(-z) A(-z)^{*}=O_{r \times s} \\
& A(z) \tilde{Q}(z)^{*}+A(-z) \tilde{Q}(-z)^{*}=H(z), \\
& \tilde{A}(z) Q(z)^{*}+\tilde{A}(-z) Q(-z)^{*}=H(z)
\end{aligned}
$$

Proof. Suppose that Equations (2.3) hold and that $H(z)$ satisfies the conditions above. Then we have

$$
\begin{aligned}
& A(z) \tilde{A}(z)^{*}+A(-z) \tilde{A}(-z)^{*} \\
& \quad=H(z) Q(z) \tilde{Q}(z)^{*} H(z)^{*}+H(-z) Q(-z) \tilde{Q}(-z)^{*} H(-z)^{*} \\
& \quad=H(z)\left[Q(z) \tilde{Q}(z)^{*}+Q(-z) \tilde{Q}(-z)^{*}\right] H(-z)^{*}=H(z) H(-z)^{*}=C I_{s \times s} .
\end{aligned}
$$

This implies that (3.3) holds. Similarly, applying Lemma 2.1, (3.4)-(3.7) can also be proven.

THEOREM 3.2. Under the condition of Lemma 3.1, suppose that $B(z)$ and $\tilde{B}(z)$ are two $s \times s$ matrices, and satisfy $B(z) \tilde{B}(z)^{*}+B(-z) \tilde{B}(-z)^{*}=(1-C) I_{s \times s}$, where $0<C<1$. Define

$$
P^{\text {new }}(z)=\left[\begin{array}{cc}
P(z) & O \\
A(z) & B(z)
\end{array}\right], \quad \tilde{P}^{\text {new }}(z)=\left[\begin{array}{cc}
\tilde{P}(z) & O \\
\tilde{A}(z) & \tilde{B}(z)
\end{array}\right] .
$$

Then $P^{\text {new }}(z) \tilde{P}^{\text {new }}(z)^{*}+P^{\text {new }}(-z) \tilde{P}^{\text {new }}(-z)^{*}=I_{(r+s) \times(r+s)}$.

ProOF. By Lemmas 2.1 and 3.1, we have

$$
\begin{array}{r}
P^{\text {new }}(z) \tilde{P}^{\text {new }}(z)^{*}+P^{\text {new }}(-z) \tilde{P}^{\text {new }}(-z)^{*} \\
=\left[\begin{array}{cc}
P(z) & 0 \\
A(z) & B(z)
\end{array}\right]\left[\begin{array}{cc}
\tilde{P}(z)^{*} & \tilde{A}(z)^{*} \\
0 & \tilde{B}(z)^{*}
\end{array}\right]
\end{array}
$$




$$
\begin{aligned}
& +\left[\begin{array}{cc}
P(-z) & 0 \\
A(-z) & B(-z)
\end{array}\right]\left[\begin{array}{cc}
\tilde{P}(-z)^{*} & \tilde{A}(-z)^{*} \\
0 & \tilde{B}(-z)^{*}
\end{array}\right] \\
= & {\left[\begin{array}{cc}
P(z) \tilde{P}(z)^{*}+P(-z) \tilde{P}(-z)^{*} & P(z) \tilde{A}(z)^{*}+P(-z) \tilde{A}(-z)^{*} \\
A(z) \tilde{P}(z)^{*}+A(-z) \tilde{P}(-z)^{*} & A(z) \tilde{A}(z)^{*}+A(-z) \tilde{A}(-z)^{*} \\
& +B(z) \tilde{B}(z)^{*}+B(-z) \tilde{B}(-z)^{*}
\end{array}\right] } \\
= & {\left[\begin{array}{ll}
I_{r \times r} & O_{r \times s} \\
O_{s \times r} & I_{s \times s}
\end{array}\right]=I_{(r+s) \times(r+s) .} }
\end{aligned}
$$

This completes the proof of Theorem 3.2.

REMARK 1. There exist a lot of $B(z), \tilde{B}(z)$ satisfying the condition

$$
B(z) \tilde{B}(z)^{*}+B(-z) \tilde{B}(-z)^{*}=(1-C) I_{s \times s}
$$

Additionally, we can choose $B(z)=\tilde{B}(z)$.

THEOREM 3.3. Suppose that all eigenvalues of the matrices $B(1)$ and $\tilde{B}(1)$ are less than 1 in modulus. If both $P(z)$ and $\tilde{P}(z)$ satisfy Condition $\mathbf{E}$, then both $P^{\text {new }}(z)$ and $\tilde{P}^{\text {new }}(z)$ satisfy Condition $\mathbf{E}$.

ProOF. Since $P^{\text {new }}(1)=\left[\begin{array}{cc}P(1) & O \\ A(1) & B(1)\end{array}\right]$, then

$$
\left|\lambda I_{(r+s) \times(r+s)}-P^{\text {new }}(1)\right|=\left|\lambda I_{r \times r}-P(1)\right|\left|\lambda I_{s \times s}-B(1)\right| \text {. }
$$

Obviously, all the eigenvalues of the matrices $P(1)$ and $B(1)$ must be the eigenvalues of the matrix $P^{\text {new }}(1)$. This means that matrix $P^{\text {new }}(1)$ has a simple eigenvalue of 1 , with all other eigenvalues less than 1 in modulus. That is, $P^{\text {new }}(z)$ satisfies Condition $\mathbf{E}$. Similarly, we can prove that $\tilde{P}^{\text {new }}(z)$ also satisfies Condition $\mathbf{E}$. This completes the proof of Theorem 3.3.

It was shown in $[7,14]$ that the representation matrix of the transition operator $\mathscr{T}_{\text {pnew }}$ is $\mathscr{T}_{\text {pnew }}=\left[2 \mathscr{A}_{2 i-j}\right]_{i, j}$, where $\mathscr{A}_{j}$ is the $(r+s)^{2} \times(r+s)^{2}$ matrix defined by $\mathscr{A}_{j}=\sum_{k} P_{k-j}^{\text {new }} \otimes P_{k}^{\text {new }}$.

According to the above discussion and [15], we have the following construction theorem.

THEOREM 3.4. Let the conditions of Lemma 3.1 and Theorems 3.2 and 3.3 be satisfied. Further, let the transition operator $\mathscr{T}_{P^{\text {new }}}$ satisfy Condition $\mathbf{E}$, and let its eigenmatrix corresponding to the eigenvalue 1 be positive definite for all $w \in R$. Then there are $\phi_{r+1}(x), \ldots, \phi_{r+s}(x)$ and $\tilde{\phi}_{r+1}(x), \ldots, \tilde{\phi}_{r+s}(x)$ such that $\Phi^{\text {new }}(x)=$ $\left[\Phi^{T}(x), \phi_{r+1}(x), \ldots, \phi_{r+s}(x)\right]^{T}$ and $\tilde{\Phi}^{\text {new }}(x)=\left[\tilde{\Phi}(x)^{T}, \tilde{\phi}_{r+1}(x), \ldots, \tilde{\phi}_{r+s}(x)\right]^{T}$ are a pair of biorthogonal multiscaling functions with multiplicity $r+s$. Their two-scale matrix symbols $P^{\text {new }}(z)$ and $\tilde{P}^{\text {new }}(z)$ are given by (3.8). 


\section{Explicit formula for constructing biorthogonal multiwavelets}

In the above section, we have given a method for constructing a pair of biorthogonal multiscaling functions. In this section, we will discuss the construction of the corresponding biorthogonal multiwavelet pair.

For simplicity, in this section, we suppose that matrices $B(z)$ and $\tilde{B}(z)$ of Theorem 3.2 satisfy the following conditions:

(A1) $B(z)=\tilde{B}(z)$;

(A2) $B(z) B(z)^{*}+B(-z) B(-z)^{*}=(1-C) I_{s \times s}$, where $0<C<1$;

(A3) $B(z) B(-z)=B(-z) B(z)$.

Clearly, if $B(z)$ is an $r \times r$ diagonal matrix, then condition (A3) must hold.

Construct the matrices $Q^{\text {new }}(z)$ and $\tilde{Q}^{\text {new }}(z)$, respectively, by

$$
\begin{aligned}
& Q^{\text {new }}(z)=\left[\begin{array}{cc}
X(z) Q(z) & Y(z) B(z) \\
O & (1-C)^{-1 / 2} z^{k} B(-z)^{*}
\end{array}\right], \\
& \tilde{Q}^{\text {new }}(z)=\left[\begin{array}{cc}
\tilde{X}(z) \tilde{Q}(z) & \tilde{Y}(z) B(z) \\
O & (1-C)^{-1 / 2} z^{k} B(-z)^{*}
\end{array}\right],
\end{aligned}
$$

where $X(z)$ and $\tilde{X}(z)$ are two $r \times r$ matrices, $Y(z)$ and $\tilde{Y}(z)$ are two $r \times s$ matrices, and $k$ is an odd number.

Next we will give an explicit formula for constructing a biorthogonal multiwavelet pair corresponding to $\Phi^{\text {new }}(x)$ and $\tilde{\Phi}^{\text {new }}(x)$.

THEOREM 4.1. Under the conditions of Theorem 3.4, if matrices $X(z), \tilde{X}(z), Y(z)$ and $\tilde{Y}(z)$ satisfy the following conditions:

$$
\left\{\begin{aligned}
H(z) X(z)^{*}+(1-C) Y(z)^{*} & =O_{s \times r} \\
H(z) \tilde{X}(z)^{*}+(1-C) \tilde{Y}(z)^{*} & =O_{s \times r} \\
X(z) \tilde{X}(z)^{*}+(1-C) Y(z) \tilde{Y}(z)^{*} & =I_{r \times r}
\end{aligned}\right.
$$

then a biorthogonal multiwavelet pair $\Psi^{\text {new }}(x)$ and $\tilde{\Psi}^{\text {new }}(x)$ corresponding to $\Phi^{\text {new }}(x)$ and $\tilde{\Phi}^{\text {new }}(x)$ is given, in terms of Fourier transforms, by

$$
\hat{\Psi}^{\text {new }}(w)=Q^{\text {new }}\left(e^{-i w / 2}\right) \hat{\Phi}^{\text {new }}(w / 2), \quad \hat{\tilde{\Psi}}^{\text {new }}(w)=\tilde{Q}^{\text {new }}\left(e^{-i w / 2}\right) \hat{\tilde{\Phi}}^{\text {new }}(w / 2) .
$$

PROOF. According to our wavelet construction theorem, we only need prove that $P^{\text {new }}(z), \tilde{P}^{\text {new }}(z), Q^{\text {new }}(z)$ and $\tilde{Q}^{\text {new }}(z)$ satisfy the following equations:

$$
\begin{aligned}
& P^{\text {new }}(z) \tilde{P}^{\text {new }}(z)^{*}+P^{\text {new }}(-z) \tilde{P}^{\text {new }}(-z)^{*}=I_{(r+s) \times(r+s)}, \\
& P^{\text {new }}(z) \tilde{Q}^{\text {new }}(z)^{*}+P^{\text {new }}(-z) \tilde{Q}^{\text {new }}(-z)^{*}=O_{(r+s) \times(r+s)}
\end{aligned}
$$




$$
\begin{aligned}
& \tilde{P}^{\text {new }}(z) Q^{\text {new }}(z)^{*}+\tilde{P}^{\text {new }}(-z) Q^{\text {new }}(-z)^{*}=O_{(r+s) \times(r+s)} \\
& Q^{\text {new }}(z) \tilde{Q}^{\text {new }}(z)^{*}+Q^{\text {new }}(-z) \tilde{Q}^{\text {new }}(-z)^{*}=I_{(r+s) \times(r+s)}
\end{aligned}
$$

By Theorem 3.2, (4.3) holds. Next, we only need to prove that (4.4), (4.5) and (4.6) hold. In fact

$$
\begin{aligned}
P^{\text {new }}(z) & \tilde{Q}^{\text {new }}(z)^{*} \\
= & {\left[\begin{array}{cc}
P(z) & 0 \\
A(z) & B(z)
\end{array}\right]\left[\begin{array}{cc}
\tilde{Q}(z)^{*} \tilde{X}(z)^{*} & O \\
B(z)^{*} \tilde{Y}(z)^{*} & (1-C)^{-1 / 2} \bar{z}^{k} B(-z)
\end{array}\right] } \\
= & \left.\begin{array}{cc}
P(z) \tilde{Q}(z)^{*} \tilde{X}(z)^{*} & O \\
A(z) \tilde{Q}(z)^{*} \tilde{X}(z)^{*}+B(z) B(z)^{*} \tilde{Y}(z)^{*} & (1-C)^{-1 / 2} \bar{z}^{k} B(z) B(-z)
\end{array}\right] .
\end{aligned}
$$

By (2.3), we have $P(z) \tilde{Q}(z)^{*}+P(-z) \tilde{Q}(-z)^{*}=O_{r \times r}$. Hence

$$
\left[P(z) \tilde{Q}(z)^{*}+P(-z) \tilde{Q}(-z)^{*}\right] \tilde{X}(z)^{*}=O_{r \times r} .
$$

Using Lemma 3.1 and the condition $B(z) B(z)^{*}+B(-z) B(-z)^{*}=(1-C) I_{s \times s}$, we obtain

$$
\begin{gathered}
{\left[A(z) \tilde{Q}(z)^{*}+A(-z) \tilde{Q}(-z)^{*}\right] \tilde{X}(z)^{*}+\left[B(z) B(z)^{*}+B(-z) B(-z)^{*}\right] \tilde{Y}(z)^{*}} \\
=H(z) \tilde{X}(z)^{*}+(1-C) \tilde{Y}(z)^{*}=O_{s \times r} .
\end{gathered}
$$

Therefore (4.4) holds. Similarly, we can prove that (4.5) holds. Finally, we prove (4.6) holds. Since

$$
\begin{aligned}
& Q^{\text {new }}(z) \tilde{Q}^{\text {new }}(z)^{*} \\
& \quad=\left[\begin{array}{cc}
X(z) Q(z) & Y(z) B(z) \\
O & (1-C)^{-1 / 2} z^{k} B(-z)^{*}
\end{array}\right]\left[\begin{array}{cc}
\tilde{Q}(z)^{*} \tilde{X}(z)^{*} & O \\
B(z)^{*} \tilde{Y}(z)^{*} & (1-C)^{-1 / 2} \bar{z}^{k} B(-z)
\end{array}\right] \\
& \quad=\left[\begin{array}{cc}
X(z) Q(z) \tilde{Q}(z)^{*} \tilde{X}(z)^{*} & (1-C)^{-1 / 2} \tilde{z}^{k} Y(z) B(z) B(-z) \\
+Y(z) B(z) B(z)^{*} \tilde{Y}(z)^{*} & \\
(1-C)^{-1 / 2} z^{k} B(-z)^{*} B(z)^{*} \tilde{Y}(z)^{*} & (1-C)^{-1} z^{k} \bar{z}^{k} B(-z)^{*} B(-z)
\end{array}\right],
\end{aligned}
$$

by (4.2), we have

$$
\begin{aligned}
Q^{\text {new }}(z) \tilde{Q}^{\text {new }}(z)^{*}+Q^{\text {new }}(-z) \tilde{Q}^{\text {new }}(-z)^{*} & \\
& =\left[\begin{array}{cc}
X(z) \tilde{X}(z)^{*}+(1-C) Y(z) \tilde{Y}(z)^{*} & O \\
O & (1-C)^{-1}\left[B(z)^{*} B(z)+B(-z)^{*} B(-z)\right]
\end{array}\right] \\
& =\left[\begin{array}{cc}
I_{r \times r} & 0 \\
O & I_{s \times s}
\end{array}\right] .
\end{aligned}
$$

This completes the proof of Theorem 4.1. 
[9] An algorithm for constructing biorthogonal multiwavelets with higher approximation orders 521

\section{Approximation orders}

In this section, we discuss the approximation orders of a pair of new biorthogonal multiscaling functions constructed in Section 3.

Let for $u=1, \ldots, s$ and $n_{u} \in \mathbb{Z}_{+}$

$$
b_{u}(z)=\sum_{j \in Z} b_{j}^{u} z^{j}=\frac{1}{2^{m-1}}\left(\frac{1+z}{2}\right)^{n_{u}} h_{u}(z), \quad h_{u}(1)=1
$$

where $h_{u}(z)$ are Laurent polynomials.

By $b_{u}(z)$ defined in (5.1), construct an $s \times s$ diagonal matrix $B(z)$ by

$$
B(z)=\operatorname{diag}\left[b_{1}(z), \ldots, b_{s}(z)\right]
$$

Then we have the following lemma.

LEMMA 5.1. Let $b_{u}(z)$ defined in (5.1) be symbols of sequences $\left\{b_{j}^{u}\right\}$. Then

$$
\begin{aligned}
2^{m} \sum_{j \in Z} b_{2 j}^{u} & =2^{m} \sum_{j \in Z} b_{2 j+1}^{u}=1, \quad u=1, \ldots, s, \\
\sum_{j \in Z}(2 j)^{k} b_{2 j}^{u} & =\sum_{j \in Z}(2 j+1)^{k} b_{2 j+1}^{u}, \quad k=1, \ldots, n_{u}-1 .
\end{aligned}
$$

Further, suppose that $B(z)=\sum_{j \in Z} B_{j} z^{j}$, and $L=\min \left\{n_{1}, \ldots, n_{s}\right\}$. Then

$$
\sum_{j \in Z}(2 j)^{k} B_{2 j}=\sum_{j \in Z}(2 j+1)^{k} B_{2 j+1}, \quad k=1, \ldots, L
$$

LEMMA 5.2. If all $b_{u}(z), u=1, \ldots, s$, satisfy $\left|b_{u}(z)\right|^{2}+\left|b_{u}(-z)\right|^{2}=2^{-(2 m-2)}$, then

$$
B(z) B(z)^{*}+B(-z) B(-z)^{*}=\left[1-\frac{2^{2 m-2}-1}{2^{2 m-2}}\right] I_{s \times s} .
$$

THEOREM 5.3. In the setting of Theorem 3.4, suppose that $\Phi(x)$ and $\tilde{\Phi}(x)$ have approximation orders $m$ and $\tilde{m}$, respectively. If the following conditions hold:

(C1) $B(z)$ given by (5.2) satisfies (5.3),

(C2) $A(z), \tilde{A}(z)$ defined in (3.1) and (3.2) satisfy

$$
A(z) \tilde{A}(z)^{*}+A(-z) \tilde{A}(-z)^{*}=\frac{2^{2 m-2}-1}{2^{2 m-2}},
$$


then $P^{\text {new }}(z)$ and $\tilde{P}^{\text {new }}(z)$ given by (3.8) can generate a pair of new biorthogonal multiscaling functions $\Phi^{\text {new }}(x)=\left[\Phi^{T}(x), \phi_{r+1}(x), \ldots, \phi_{r+s}(x)\right]^{T}$ and $\tilde{\Phi}^{\text {new }}(x)=$ $\left[\tilde{\Phi}(x)^{T}, \tilde{\phi}_{r+1}(x), \ldots, \tilde{\phi}_{r+s}(x)\right]^{T}$, which have approximation orders $m+L$ and $\tilde{m}+L$, respectively.

Proof. By Theorem 3.4, $P^{\text {new }}(z)$ and $\tilde{P}^{\text {new }}(z)$ can generate a new biorthogonal multiscaling function pair $\Phi^{\text {new }}(x)$ and $\tilde{\Phi}^{\text {new }}(x)$. Next, we will prove that this new biorthogonal multiscaling function pair have approximation orders of $m+L$ and $\tilde{m}+L$, respectively.

Since the approximation order of $\Phi(x)$ is $m$, there are $\mathbf{a}^{\ell} \in R^{r}, \ell=0,1, \ldots, m-1$, with $\mathbf{a}^{0} \neq O_{1 \times r}$, such that, by (2.4) and (2.5),

$$
\begin{aligned}
\mathbf{a}^{\ell}\left(\sum_{j \in Z} P_{2 j}-\frac{1}{2^{\ell}} I_{r \times r}\right) & =-\sum_{k=0}^{\ell-1}(-1)^{\ell-k} \frac{1}{2^{\ell-k}}\left(\begin{array}{l}
\ell \\
k
\end{array}\right) \mathbf{a}^{k} \sum_{j \in Z}(2 j)^{\ell-k} P_{2 j}, \\
\mathbf{a}^{\ell}\left(\sum_{j \in Z} P_{2 j+1}-\frac{1}{2^{\ell}} I_{r \times r}\right) & =-\sum_{k=0}^{\ell-1}(-1)^{\ell-k} \frac{1}{2^{\ell-k}}\left(\begin{array}{l}
\ell \\
k
\end{array}\right) \mathbf{a}^{k} \sum_{j \in Z}(2 j+1)^{\ell-k} P_{2 j+1} .
\end{aligned}
$$

Next, we will prove the approximation order of $\Phi^{\text {new }}(x)$ is $m+L$. That is, we will find a set of row vectors $\mathbf{w}^{\ell} \in R^{r+s}, \ell=0,1, \ldots, m+L-1$, with $\mathbf{w}^{0} \neq O_{1 \times(r+s)}$ such that

$$
\begin{aligned}
& \mathbf{w}^{\ell}\left(\left[\begin{array}{cc}
\sum_{j \in Z} P_{2 j} & O_{r \times s} \\
\sum_{j \in Z} A_{2 j} & \sum_{j \in Z} B_{2 j}
\end{array}\right]-\frac{1}{2^{\ell}} I_{(r+s) \times(r+s)}\right) \\
& =-\sum_{k=0}^{\ell-1} \frac{(-1)^{\ell-k}}{2^{\ell-k}}\left(\begin{array}{l}
\ell \\
k
\end{array}\right) \mathbf{w}^{k}\left[\begin{array}{cc}
\sum_{j \in Z}(2 j)^{\ell-k} P_{2 j} & O_{r \times s} \\
\sum_{j \in Z}(2 j)^{\ell-k} A_{2 j} & \sum_{j \in Z}(2 j)^{\ell-k} B_{2 j}
\end{array}\right] \\
& \mathbf{w}^{\ell}\left(\left[\begin{array}{cc}
\sum_{j \in Z} P_{2 j+1} & O_{r \times s} \\
\sum_{j \in Z} A_{2 j+1} & \sum_{j \in Z} B_{2 j+1}
\end{array}\right]-\frac{1}{2^{\ell}} I_{(r+s) \times(r+s)}\right) \\
& =-\sum_{k=0}^{\ell-1} \frac{(-1)^{\ell-k}}{2^{\ell-k}}\left(\begin{array}{l}
\ell \\
k
\end{array}\right) \mathbf{w}^{k}\left[\begin{array}{cc}
\sum_{j \in Z}(2 j+1)^{\ell-k} P_{2 j+1} & O_{r \times s} \\
\sum_{j \in Z}(2 j+1)^{\ell-k} A_{2 j+1} & \sum_{j \in Z}(2 j+1)^{\ell-k} B_{2 j+1}
\end{array}\right] .
\end{aligned}
$$

It is clear that $\mathbf{w}^{\ell}=\left[\mathbf{a}^{\ell}, 0, \ldots, 0\right] \in R^{r+s}, \ell=0,1, \ldots, m-1$, as the first $m$ vectors satisfy (5.4) and (5.5). Hence we choose $\mathbf{w}^{\ell}=\left[\mathbf{a}^{\ell}, 0, \ldots, 0\right] \in R^{r+s}$, $\ell=0,1, \ldots, m-1$, to be the first $m$ vectors in (5.4) and (5.5). The remaining $L$ row vectors are denoted by $\mathbf{w}^{m+\ell}=\left[\mathbf{a}^{m+\ell}, c_{m+\ell}^{1}, c_{m+\ell}^{2}, \ldots, c_{m+\ell}^{s}\right], \ell=0,1, \ldots, L-1$. Obviously, $\mathbf{w}^{m}$ must satisfy $\sum_{j=1}^{s}\left|c_{m}^{j}\right| \neq 0$. In fact, if all $c_{m}^{j}=0$, then $\mathbf{w}^{m}=$ $\left[\mathbf{a}^{m}, 0, \ldots, 0\right]$. This means that the approximation order of $\Phi(x)$ is $m+1$. If we use the notation $\mathbf{w}^{\ell}=\left[\mathbf{a}^{\ell}, c_{\ell}^{1}, c_{\ell}^{2}, \ldots, c_{\ell}^{s}\right]$, then $c_{\ell}^{j}=0$ for $j=1, \ldots, s ; \ell=0,1, \ldots, m-1$. 
Hence (5.4) is equivalent to

$$
\begin{aligned}
& \mathbf{a}^{\ell}\left(\sum_{j \in Z} P_{2 j}-\frac{1}{2^{\ell}} I_{r \times r}\right)+\left[c_{\ell}^{1}, \ldots, c_{\ell}^{s}\right] \sum_{j \in Z} A_{2 j} \\
& \quad=-\sum_{k=0}^{\ell-1} \frac{(-1)^{\ell-k}}{2^{\ell-k}}\left(\begin{array}{l}
\ell \\
k
\end{array}\right)\left[\mathbf{a}^{k} \sum_{j \in Z}(2 j)^{\ell-k} P_{2 j}+\left[c_{k}^{1}, \ldots, c_{k}^{s}\right] \sum_{j \in Z}(2 j)^{\ell-k} A_{2 j}\right], \\
& {\left[c_{\ell}^{1}, \ldots, c_{\ell}^{s}\right]\left[\sum_{j \in Z} B_{2 j}-\frac{1}{2^{\ell}} I_{s \times s}\right]} \\
& \quad=-\sum_{k=0}^{\ell-1} \frac{(-1)^{\ell-k}}{2^{\ell-k}}\left(\begin{array}{l}
\ell \\
k
\end{array}\right)\left[c_{k}^{1}, \ldots, c_{k}^{s}\right] \sum_{j \in Z}(2 j)^{\ell-k} B_{2 j} .
\end{aligned}
$$

Since $c_{\ell}^{j}=0$ for $j=1, \ldots, s, \ell=0,1, \ldots, m-1$, then (5.7) implies the following two identities:

$$
\begin{aligned}
& {\left[c_{m}^{1}, \ldots, c_{m}^{s}\right]\left[\sum_{j \in Z} B_{2 j}-\frac{1}{2^{m}} I_{s \times s}\right]=O_{s \times s},} \\
& {\left[c_{m+\ell}^{1}, \ldots, c_{m+\ell}^{s}\right]\left[\sum_{j \in Z} B_{2 j}-\frac{1}{2^{m+\ell}} I_{s \times s}\right]} \\
& \quad=-\sum_{k=0}^{\ell-1} \frac{(-1)^{\ell-k}}{2^{\ell-k}}\left(\begin{array}{c}
m+\ell \\
\ell-k
\end{array}\right)\left[c_{m+k}^{1}, \ldots, c_{m+k}^{s}\right] \sum_{j \in Z}(2 j)^{\ell-k} B_{2 j},
\end{aligned}
$$

for $\ell=1, \ldots, L-1$.

By Lemma 5.1, $\sum_{j \in Z} B_{2 j}=2^{-m} I_{s \times s}$. Hence

$$
\sum_{j \in Z} B_{2 j}-\frac{1}{2^{m+\ell}} I_{s \times s}=\frac{2^{\ell}-1}{2^{m+\ell}} I_{s \times s} .
$$

Therefore, for $\ell=1, \ldots, L-1$,

$$
\begin{aligned}
& {\left[c_{m+\ell}^{1}, \ldots, c_{m+\ell}^{s}\right]} \\
& \quad=-\frac{2^{m}}{2^{\ell}-1} \sum_{k=0}^{\ell-1}(-1)^{\ell-k} 2^{k}\left(\begin{array}{c}
m+\ell \\
\ell-k
\end{array}\right)\left[c_{m+k}^{1}, \ldots, c_{m+k}^{s}\right] \sum_{j \in Z}(2 j)^{\ell-k} B_{2 j} .
\end{aligned}
$$

Similarly, applying (5.5), we have

$$
\left[c_{m}^{1}, \ldots, c_{m}^{s}\right]\left[\sum_{j \in Z} B_{2 j+1}-\frac{1}{2^{m}} I_{s \times s}\right]=O_{s \times s},
$$




$$
\begin{aligned}
& {\left[c_{m+\ell}^{1}, \ldots, c_{m+\ell}^{s}\right]\left[\sum_{j \in Z} B_{2 j+1}-\frac{1}{2^{m+\ell}} I_{s \times s} \cdot\right]} \\
& \quad=-\sum_{k=0}^{\ell-1} \frac{(-1)^{\ell-k}}{2^{\ell-k}}\left(\begin{array}{c}
m+\ell \\
\ell-k
\end{array}\right)\left[c_{m+k}^{1}, \ldots, c_{m+k}^{s}\right] \sum_{j \in Z}(2 j+1)^{\ell-k} B_{2 j+1},
\end{aligned}
$$

for $\ell=1, \ldots, L-1$. Hence we have

$$
\begin{aligned}
{\left[c_{m+\ell}^{1}, \ldots, c_{m+\ell}^{s}\right]=} & -\frac{2^{m}}{2^{\ell}-1} \sum_{k=0}^{\ell-1}(-1)^{\ell-k} 2^{k}\left(\begin{array}{c}
m+\ell \\
\ell-k
\end{array}\right) \\
& \times\left[c_{m+k}^{1}, \ldots, c_{m+k}^{s}\right] \sum_{j \in Z}(2 j+1)^{\ell-k} B_{2 j+1},
\end{aligned}
$$

for $\ell=1, \ldots, L-1$. By (5.10) or (5.11), taking any $\left[c_{m}^{1}, \ldots, c_{m}^{s}\right] \neq \mathbf{O}_{1 \times s}$, we can obtain $\left[c_{m+\ell}^{1}, \ldots, c_{m+\ell}^{s}\right], \ell=1, \ldots, L-1$. And then applying (5.6), we can obtain $\mathbf{a}^{m+\ell}$. This means that the remaining $L-1$ row vectors $\mathbf{w}^{m+\ell}=\left[\mathbf{a}^{m+\ell}, c_{m+\ell}^{1}, \ldots, c_{m+\ell}^{s}\right]$, $\ell=1, \ldots, L-1$ are obtained. Thereby, we prove that $\Phi^{\text {new }}(x)$ has approximation order $m+L$. Similarly, we also prove that the approximation order of $\tilde{\Phi}^{\text {new }}(x)$ is $\tilde{m}+L$. This completes the proof of Theorem 5.3.

REMARK 2. Lemma 5.1 can guarantee that vectors $\left[c_{m+\ell}^{1}, \ldots, c_{m+\ell}^{s}\right], \ell=1, \ldots$, $L-1$, obtained by $(5.10)$ and $(5.11)$ are the same.

\section{Example}

Case of $r=s=1$ Let $\phi_{1}(x)$ and $\tilde{\phi}_{1}(x)$ be a pair of biorthogonal scaling functions, and let $\psi_{1}(x)$ and $\tilde{\psi}_{1}(x)$ be the corresponding biorthogonal wavelet pair. Their corresponding two-scale symbols are

$$
\begin{array}{lll}
P(z)=\left[\frac{1+z}{2}\right]^{2}\left(-\frac{1}{2} z^{-2}+2 z^{-1}-\frac{1}{2}\right), & \tilde{P}(z)=\left[\frac{1+z}{2}\right]^{2} z^{-1}, \\
Q(z)=-\frac{1}{4} z^{2}+\frac{1}{2} z-\frac{1}{4} & \text { and } & \tilde{Q}(z)=-\frac{1}{8} z^{3}-\frac{1}{4} z^{2}+\frac{3}{4} z-\frac{1}{4}-\frac{1}{8} z^{-1} .
\end{array}
$$

It is easy to verify that both the approximation orders of $\phi(x)$ and $\tilde{\phi}(x)$ are 2 . That is, $m=\tilde{m}=2$. Take

$$
H(z)=\sqrt{\frac{2^{2 m-2}-1}{2^{2 m-2}}}=\frac{\sqrt{3}}{2} .
$$

Then by (3.1) and (3.2), $A(z)=(\sqrt{3} / 2) Q(z)$ and $\tilde{A}(z)=(\sqrt{3} / 2) \tilde{Q}(z)$. Take

$$
B(z)=\frac{1}{2}\left[\frac{1+z}{2}\right]^{2} \frac{(1+\sqrt{3})+(1-\sqrt{3}) z}{2} \text {. }
$$


It is easy to verify that

$$
A(z) \widetilde{A}(z)^{*}+A(-z) \tilde{A}(-z)^{*}=3 / 4, \quad B(z) B(z)^{*}+B(-z) B(-z)^{*}=1-3 / 4 .
$$

By (3.8), we construct

$$
\begin{aligned}
& P^{\text {new }}(z)=\left[\begin{array}{cc}
{\left[\frac{1+z}{2}\right]^{2}\left(-\frac{1}{2} z^{-2}+2 z^{-1}-\frac{1}{2}\right)} & 0 \\
\frac{\sqrt{3}}{2}\left(-\frac{1}{4} z^{2}+\frac{1}{2} z-\frac{1}{4}\right) & \frac{1}{2}\left[\frac{1+z}{2}\right]^{2} \\
\frac{(1+\sqrt{3})+(1-\sqrt{3}) z}{2}
\end{array}\right], \\
& \tilde{P}^{\text {new }}(z)=\left[\begin{array}{cc}
{\left[\frac{1+z}{2}\right]^{2} z^{-1}} & 0 \\
\frac{\sqrt{3}}{2}\left(-\frac{1}{8} z^{3}-\frac{1}{4} z^{2}+\frac{3}{4} z-\frac{1}{4}-\frac{1}{8} z^{-1}\right) & \frac{1}{2}\left[\frac{1+z}{2}\right]^{2} \frac{(1+\sqrt{3})+(1-\sqrt{3}) z}{2}
\end{array}\right] .
\end{aligned}
$$

From [6, 14], the transition operation $\mathscr{T}_{\text {pew }}$ associated with $P^{\text {new }}(z)$ is a $44 \times 44$ matrix. By calculation, the transition operation $\mathscr{T}_{\text {prew }}$ satisfies condition $\mathbf{E}$. Hence, applying Theorem 3.4, we obtain a pair of new biorthogonal multiscaling functions $\Phi^{\text {new }}(x)=\left[\phi_{1}(x), \phi_{2}(x)\right]^{T}$ and $\tilde{\Phi}^{\text {new }}(x)=\left[\tilde{\phi}_{1}(x), \tilde{\phi}_{2}(x)\right]^{T}$, with two-scale matrix symbols $P^{\text {new }}(z)$ and $\tilde{P}^{\text {new }}(z)$ given by $(6.1)$ and $(6.2)$, respectively.

Let $X(z)=X(z)^{*}=1 / 2$ and $Y(z)=Y(z)^{*}=-\sqrt{3}$. It is easy to verify that $X(z), X(z)^{*}, Y(z)$ and $Y(z)^{*}$ satisfy (4.2). Thus, by (4.1), and taking $k=3$, we can construct two matrices $Q^{\text {new }}(z)$ and $\tilde{Q}^{\text {new }}(z)$. Hence, applying Theorem 4.1, the corresponding biorthogonal multiwavelet pair $\Psi^{\text {new }}(x)=\left[\psi_{1}(x), \psi_{2}(x)\right]^{T}$ and $\tilde{\Psi}^{\text {new }}(x)=\left[\tilde{\psi}_{1}(x), \tilde{\psi}_{2}(x)\right]^{T}$ can be constructed by the two scale matrix symbols $Q^{\text {new }}(z)$ and $\tilde{Q}^{\text {new }}(z)$.

Further, by Theorem 5.3, both approximation orders of the new biorthogonal multiscaling functions $\Phi^{\text {new }}(x)$ and $\tilde{\Phi}^{\text {new }}(x)$ are 4 . That is, we raise the approximation orders of $\phi_{1}(x)$ and $\tilde{\phi}_{1}(x)$ from 2 to 4 .

Similar to the case of $r=s=1$, some examples can also be constructed for the settings $r>1$ and $s>1$.

\section{Acknowledgements}

The author wishes to thank the referees for their valuable suggestions and comments which have improved the presentation of the results.

This research was supported by the Natural Science Foundation of Guangdong Province (Grant Nos. 05008289 and 032038), and the Doctoral Foundation of Guangdong Province (Grant No. 04300917).

\section{References}

[1] C. K. Chui and J.-A. Lian, "A study on orthonormal multi-wavelets", Appl. Numer. Math. 20(1996) 273-298. 
[2] C. K. Chui and J.-A. Lian, "Construction of orthonormal multi-wavelets with additional vanishing moments", Adv. Comput. Math. 24 (2006) 239-262.

[3] A. Cohen, I. Daubechies and G. Plonka, "Regularity of refinable function vectors", J. Fourier Anal. Appl. 3 (1997) 295-324.

[4] W. Dahmen and C. A. Micchelli, "Biorthogonal wavelet expansion", Constr. Approx. 13 (1997) 293-328.

[5] C. Heil and D. Collela, "Matrix refinement equations: Existence and uniqueness", J. Fourier Anal. Appl. 2 (1996) 363-377.

[6] Q. Jiang, "On the regularity of matrix refinable functions", SIAM J. Math. Anal. Appl. 29 (1998) 1157-1176.

[7] Q. Jiang, “Orthogonal multiwavelets with optimum time-frequency resolution”, IEEE Trans. Signal Process. 46 (1998) 830-844.

[8] J.-A. Lian, "On the order of polynomial reproduction for multi-scaling functions", Appl. Comp. Harm. Anal. 3 (1996) 358-365.

[9] J.-A. Lian, "Orthogonal criteria for multiscaling functions", Appl. Comput. Harmon. Anal. 5 (1998) 277-311.

[10] J.-A. Lian and C. K. Chui, "Analysis-ready multiwavelets (armlets) for processing scalar-valued signals", IEEE Trans. Signal Process. Lett. 11 (2004) 205-208.

[11] J.-A. Lian and C. K. Chui, "Balanced multiwavelets with short filters", IEEE Trans. Signal Process. Lett. 11 (2004) 75-78.

[12] G. Plonka, "Approximation order provided by refinable function vectors", Constr. Approx. 13 (1997) 221-244.

[13] G. Plonka and V. Strela, "Construction of multiscaling functions with approximation and symmetry", SIAM J. Math. Anal. Appl. 29 (1998) 481-510.

[14] G. Plonka and V. Strela. "From wavelets to multiwavelets", in Mathematical methods for curves and surfaces, II (eds. M. Daehlen, T. Lyche and L. L. Schumaker), (Vanderbilt Univ. Press, Nashville, TN, 1998) 1-25.

[15] Z. Shen, "Refinable function vectors", SIAM J. Math. Anal. Appl. 29 (1998) 235-250.

[16] V. Strela, "Multiwavelets: Regularity, orthogonality and symmetry via two-scale similarity transform", Studies in Appl. Math. 98 (1997) 335-354.

[17] S.-Z. Yang, "A fast algorithm for constructing orthogonal multiwavelets", ANZIAM J. 46. (2004) 185-202.

[18] S.-Z. Yang and Z.-X. Cheng. "Orthonormal multi-wavelets on the interval [0,1] with multiplicity $r "$, Acta Mathematica Sinica 45 (2002) 789-796.

[19] S.-Z. Yang, Z.-X. Cheng and H.-Y. Wang, "Construction of biorthogonal multiwavelets", J. Math. Anal. Appl. 276 (2002) 1-12. 